\title{
The Clinical Characteristics of Two Anti-OJ (Anti-Isoleucyl- tRNA Synthetase) Autoantibody-Positive Interstitial Lung Disease Patients with Polymyositis/Dermatomyositis
}

\author{
Kei Kunimasa ${ }^{1,8}$, Machiko Arita ${ }^{1}$, Takashi Nakazawa ${ }^{2}$, Maki Tanaka ${ }^{1}$, Kazuya Tsubouchi ${ }^{1,9}$, \\ Satoshi Konishi ${ }^{1,10}$, Yasushi Fukuda ${ }^{1}$, Mika Saigusa ${ }^{1,3}$, Hiroaki Nakagawa ${ }^{1,4}$, \\ Satoshi Ubukata ${ }^{1,5}$, Yohei Korogi ${ }^{1}$, Takao Fujii ${ }^{6}$, Tsuneyo Mimori ${ }^{7}$ and Tadashi Ishida ${ }^{1}$
}

\begin{abstract}
We herein report the clinical and laboratory characteristics of two anti-OJ (anti-isoleucyl-tRNA synthetase) autoantibody-positive interstitial lung disease patients with polymyositis/dermatomyositis (PM/DM). We compared these characteristics with previously published findings. Previous reports and our present cases show that anti-OJ autoantibody-positive interstitial lung disease (ILD) patients with PM/DM lack the manifestations of Raynaud's phenomenon and sclerodactyly and show good prognoses and responses to glucocorticoid therapy. These results indicate that the presence of anti-OJ autoantibodies may be useful for predicting the prognosis of ILD and its clinical course in PM/DM patients.
\end{abstract}

Key words: interstitial lung disease, polymyositis/dermatomyositis, anti-aminoacyl-tRNA antibodies

(Intern Med 51: 3405-3410, 2012)

(DOI: 10.2169/internalmedicine.51.7452)

\section{Introduction}

Anti-aminoacyl-tRNA synthetase (anti-ARS) autoantibodies are generally found in patients with polymyositis (PM) and dermatomyositis (DM), particularly in those with associated interstitial lung disease (ILD) (1). Autoantibodies directed against aminoacyl-tRNA synthetases are found in approximately $25 \%$ to $35 \%$ of patients with PM and DM (2). ARS comprises a family of cytoplasmic enzymes that catalyze the formation of aminoacyl-tRNA from a specific amino acid and its cognate tRNA and play a crucial role in protein synthesis (3). Eight classes of autoantibodies that react with ARS have been recognized: anti-histidyl (anti-Jo-1), anti-threonyl (anti-PL-7), anti-alanyl (anti-PL-12), anti- glycyl (anti-EJ), anti-isoleucyl (anti-OJ), anti-asparaginyl (anti-KS), anti-phenylalanyl (anti-Zo) and anti-tyrosyl-tRNA (anti-YRS) synthetases (4-6). Anti-Jo-1 antibodies were the first of these antibodies to be discovered and are detectable in approximately $20 \%$ to $30 \%$ of PM/DM patients (7). AntiOJ antibodies appear to be associated with clinical manifestations that are generally similar to those of other antisynthetases, including myositis, ILD and Raynaud's phenomenon. In previous studies, anti-OJ antibodies were found in fewer than $2 \%$ of all patients with PM/DM (8). Sato et al. (9) reported the clinical characteristics of seven Japanese patients with anti-OJ autoantibodies. These patients lacked any manifestations of Raynaud's phenomenon or sclerodactyly and suffered from ILD associated with PM. This group showed that anti-OJ antibodies are a clinically important

\footnotetext{
${ }^{1}$ Department of Respiratory Medicine, Kurashiki Central Hospital, Japan, ${ }^{2}$ Department of Endocrinology and Rheumatology, Kurashiki Central Hospital, Japan, ${ }^{3}$ Department of Palliative Medicine, Okayama Saiseikai General Hospital, Japan, ${ }^{4}$ Department of Respiratory Medicine, Shiga University of Medical Science, Japan, ${ }^{5}$ Department of Respiratory Diseases, Saka General Hospital, Japan, ${ }^{6}$ Department of the Control for Rheumatic Diseases, Graduate School of Medicine, Kyoto University, Japan, ${ }^{7}$ Department of Rheumatology and Clinical Immunology, Graduate School of Medicine, Kyoto University, Japan, ${ }^{8}$ Division of Genetics, Kobe University Graduate School of Medicine, Japan, ${ }^{9}$ Department of Respiratory Medicine, St. Mary's Hospital, Japan and ${ }^{10}$ Department of Respiratory Medicine, Graduate School of Medicine, Kyoto University, Japan Received for publication January 31, 2012; Accepted for publication September 10, 2012

Correspondence to Dr. Kei Kunimasa, keikunimasa@gmail.com
} 
Table 1. Laboratory and Radiological Data

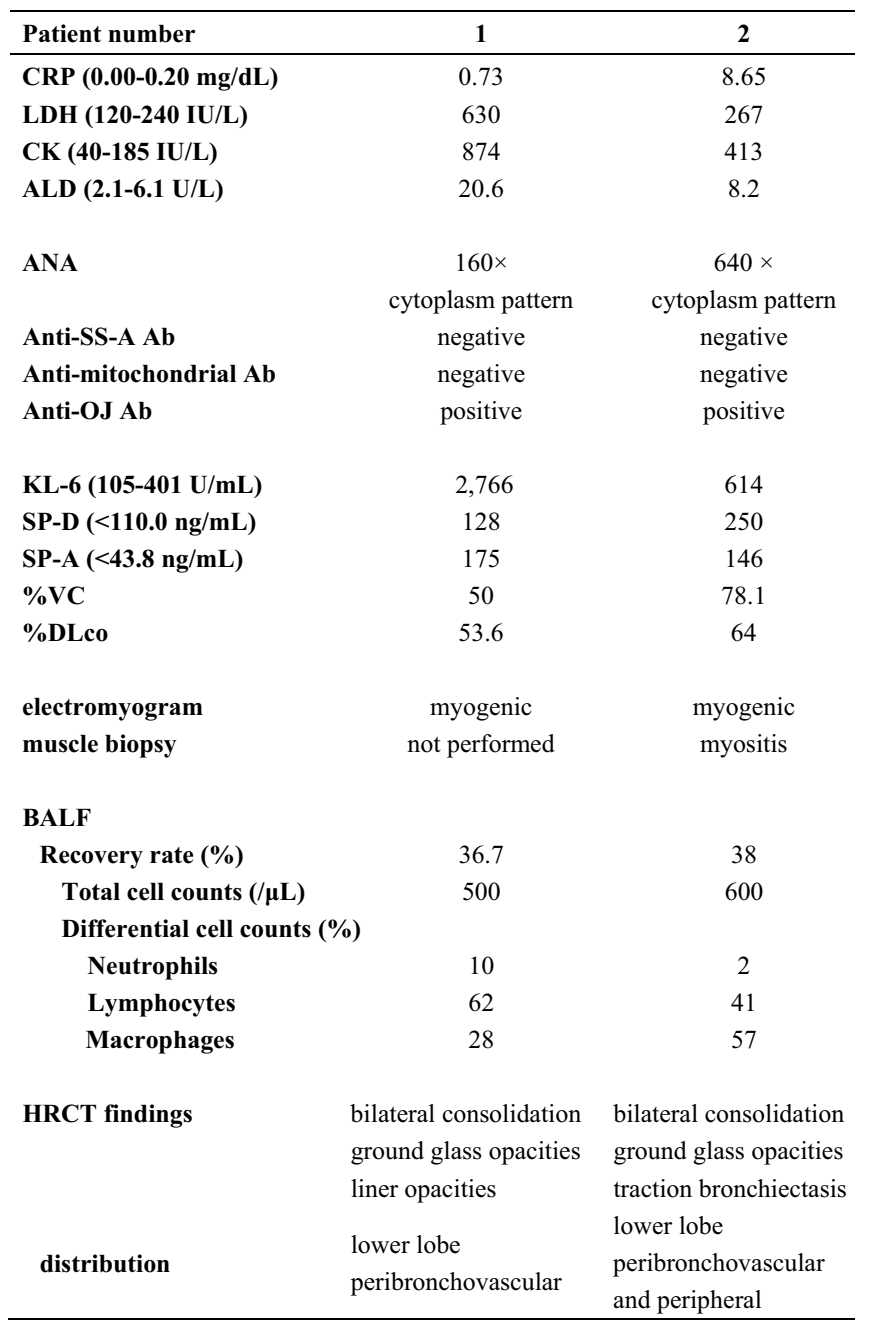

marker of a specific subset of anti-ARS syndromes. However, there are still very few reports regarding the clinical characteristics of PM/DM patients with anti-OJ autoantibodies; therefore, collecting cases to identify the clinical features is necessary. We herein present the clinical characteristics of two anti-OJ autoantibody-positive ILD patients with $\mathrm{PM} / \mathrm{DM}$

\section{Case Reports}

\section{Case 1}

A 67-year-old woman was admitted to our hospital with a 2-month history of dry cough, slight fever and worsening dyspnea on exertion. She also complained of mild myalgia in both thighs. One month earlier, an abnormal chest roentgenogram was found, and she was referred to our hospital for extensive testing. She had a history of diabetes mellitus, which was treated with diet therapy, and carpal tunnel syndrome. She was on no medications. She had never smoked and had no history of alcohol use or pet ownership. She had a family history of diabetes mellitus and liver disease; however, she did not have a family history of connective tissue disease (CTD), rheumatic diseases, collagen vascular dis-
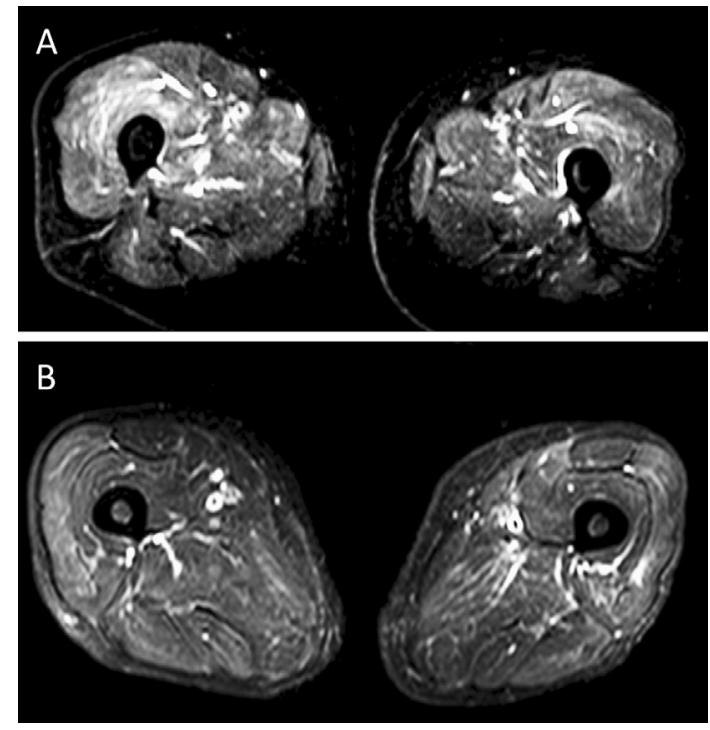

Figure 1. Lower limb muscle MRI in three patients. Fatsuppressed T2-weighted imaging in Case 1 showing (A) diffuse, abnormally high signals in the bilateral quadriceps and in Case 2 showing (B) diffuse, abnormally high signals in the bilateral quadriceps.

eases or systemic autoimmune diseases. She lived with her husband in an old wooden house that had been standing for 40 years.

A physical examination showed scaly erythema on the dorsum of the hands (Gottron's sign), mechanic's hand and spotty hemorrhages in some nail fold capillaries. Chest auscultation revealed fine crackles in the lower aspects of both lungs. On neurological examination, the patient's Manual Muscle Test (MMT) scores were 4 in the infraspinatus and iliopsoas muscles and 5 in the other muscles. The findings of other neurological assessments were normal, including those of the cranial nerves, sensory function and tendon reflexes. The laboratory findings and results of the pulmonary function tests are shown in Table 1. Magnetic resonance imaging (MRI) of the bilateral thighs showed diffuse, abnormally high T2-weighted imaging (T2WI) signals (Fig. 1A). Findings from needle electromyography of the right quadriceps indicated the presence of myogenic changes with concomitant active denervation. Chest X-ray and high-resolution computed tomography (HRCT) findings of the chest are shown in Figs. 2A, 3A, 3B. HRCT showed bilateral consolidation predominantly distributed along the bronchovascular bundles in both lower lobes and ground glass attenuation. Bronchoalveolar lavage (BAL) evaluation was performed (Table 1), which showed lymphocyte predominance. A Gram stain of the BAL fluid (BALF) was negative. It was difficult to perform a histopathological examination of the muscles or lungs due to deterioration of the patient's respiratory symptoms. These findings led us to diagnose the patient with interstitial pneumonia associated with DM. A fluorescent antinuclear antibody test was positive and showed a cytoplasmic pattern. An anti-Jo-1 antibody test was negative; therefore, we assayed other anti-ARS autoantibodies with an 

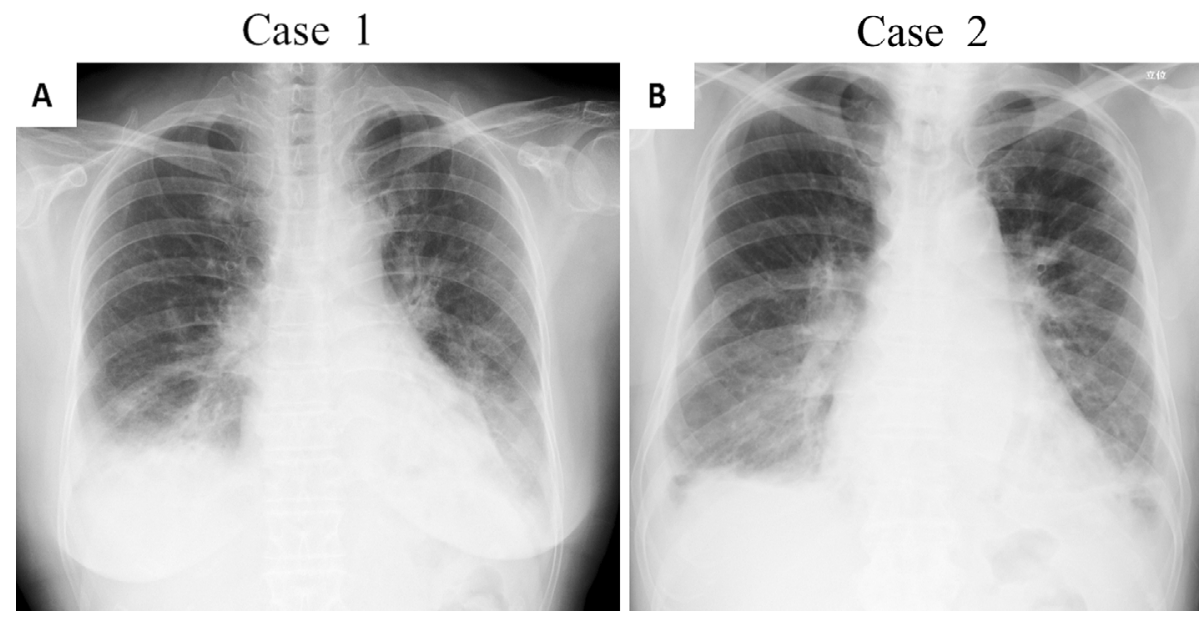

Figure 2. Chest radiographs. Case 1: (A) bilateral consolidation and ground glass attenuation with volume loss in both lower lobes; Case 2: (B) bilateral consolidation and ground glass attenuation with volume loss in both lower lobes.

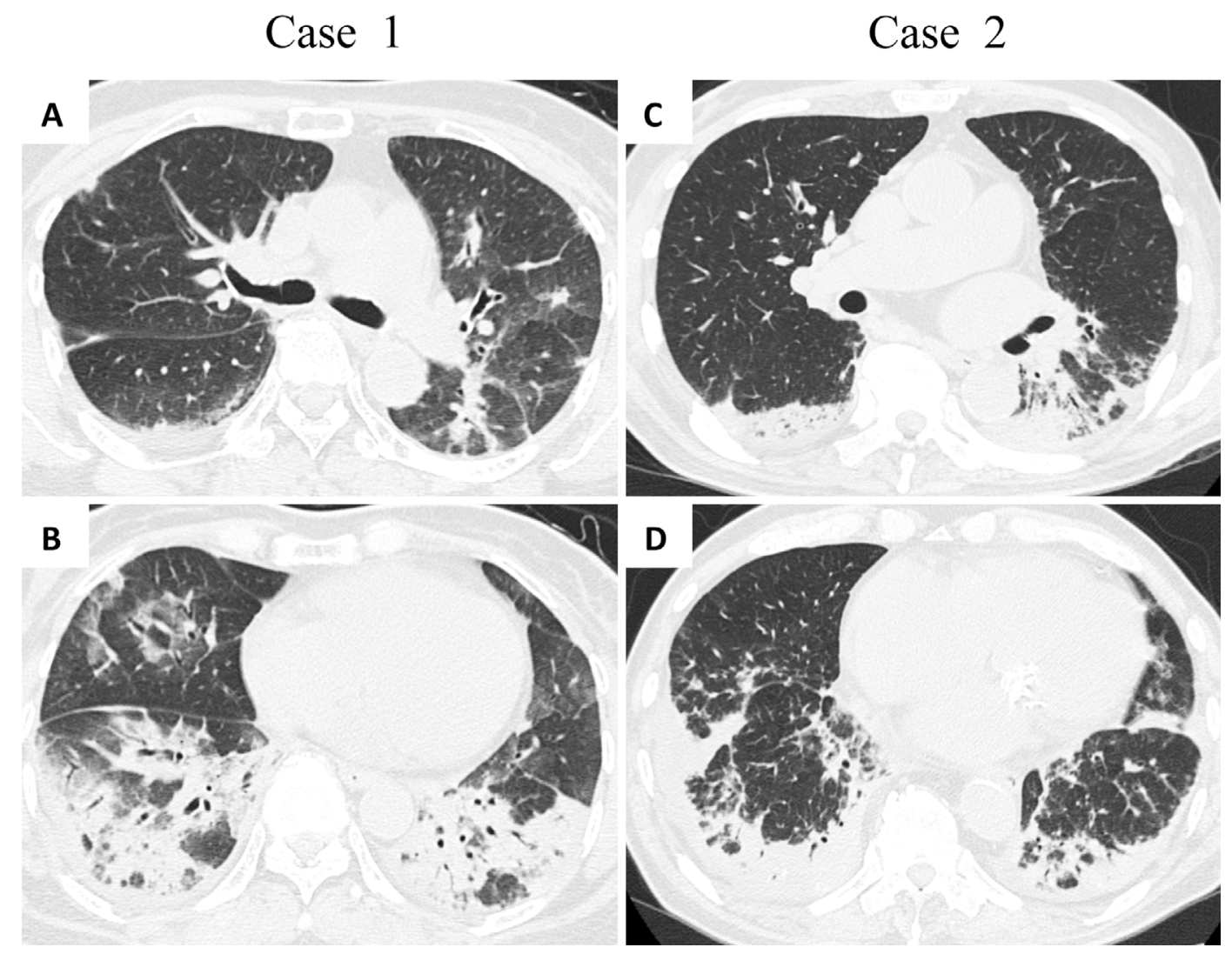

Figure 3. High-resolution chest CT scans. Case 1: (A, B) bilateral consolidation predominantly distributed along the bronchovascular bundles with volume loss in both lower lobes and areas of ground glass attenuation and liner opacities; Case 2: (C, D) bilateral consolidation predominantly distributed in peripheral areas with volume loss in both lower lobes and areas of ground glass attenuation, intralobular reticular opacities and traction bronchiectasis.

RNA immunoprecipitation assay and found that the anti-OJ antibody test was positive. The patient was treated with high-dose methylprednisolone (1 g/day intravenously for three days) followed by oral prednisolone $(1 \mathrm{mg} / \mathrm{kg} / \mathrm{day})$ and cyclosporine $(5 \mathrm{mg} / \mathrm{kg} /$ day $)$. Her clinical condition and chest radiography findings showed remarkable improvements after
12 months of treatment.

\section{Case 2}

A 74-year-old man was admitted to our hospital with a 1month history of fever, fatigue and worsening dyspnea on exertion. Two weeks prior to admission, he noticed a pro- 
Table 2. Clinical Characteristics

\begin{tabular}{lcc}
\hline \multicolumn{1}{c}{ Patient number } & $\mathbf{1}$ & $\mathbf{2}$ \\
\hline Gender & female & male \\
Age & 67 & 74 \\
Diagnosis & $\mathrm{DM}$ & $\mathrm{PM}$ \\
initial symptoms & & \\
cough & + & + \\
dyspnea & + & + \\
arthritis & - & - \\
weight loss & - & - \\
muscle weakness & + & - \\
fever & - & + \\
myositis & + & + \\
Rash & + & - \\
Raynaud's phenomenon & - & - \\
abnormal capillaries of nail folds & & + \\
$\quad$ micro hemorrhage & + & - \\
$\quad$ enlarged capillary & - & - \\
sicca & - & - \\
mechanic's hand & Gottron sign $(+)$ & \\
treatment & PSL pulse $\rightarrow 1.0 \mathrm{mg} / \mathrm{kg}$ & PSL $1.0 \mathrm{mg} / \mathrm{kg}$ \\
effect & Improved & \\
\hline
\end{tabular}

ductive cough and was seen at the nearest clinic. He was diagnosed with bronchitis and treated with levofloxacin and a combination cold remedy. However, his clinical condition did not improve and instead gradually deteriorated. Therefore, he was referred to our hospital for extensive testing. He had a history of atrial fibrillation, mitral regurgitation, mitral stenosis and diabetes mellitus and had been hospitalized seven years earlier for approximately three months with infectious endocarditis caused by Group A Streptococcus. His medications included metildigoxin, fulosemide, warfarin, amlodipine, candesartan and glimepiride. He had never smoked and had no history of alcohol use or pet ownership. He had no family history of collagen or muscle disease.

On physical examination, he was found to have cornification, but no erythema, spread in a symmetric fashion over the metacarpophalangeal joints along with spotty hemorrhages in some nail fold capillaries; however, no muscle weakness was observed. Cardiac auscultation revealed a systolic murmur (Levine III/VI), and chest auscultation showed fine crackles in the lower aspects of both lungs. On neurological examination, the MMT scores were full in all muscles. The findings of other neurological assessments were normal. The laboratory findings and results of the pulmonary function tests are shown in Table 1. Although the patient did not show any muscle symptoms, an MRI study of the bilateral thighs showed diffuse, abnormally high T2-WI signals (Fig. 1B). Findings from needle electromyography of the right quadriceps indicated the presence of myogenic changes with concomitant active denervation, and a muscle biopsy of the left quadriceps revealed myositis. The findings of chest X-ray and HRCT of the chest are shown in Figs. 2B, 3C, 3D. HRCT showed bilateral consolidation predominantly distributed along the bronchovascular bundles in both lower lobes and ground glass attenuation with traction bronchiectasis. A BAL analysis was performed (Table 1), which showed lymphocyte predominance. These findings led us to diagnose the patient with interstitial pneumonia associated with PM. Later, an anti-OJ antibody test was shown to be positive. The patient was treated with oral prednisolone $(1 \mathrm{mg} / \mathrm{kg} / \mathrm{day})$. His clinical condition and chest radiography findings showed remarkable improvements, and no deteriorations were observed over a 3 -year treatment period.

\section{Discussion}

Previous studies have indicated that ILD is associated with high mortality in PM/DM patients. ILD occurs in as many as $53 \%$ of PM/DM patients (10) and may lead to life threatening complications, including ventilator failure and secondary pulmonary hypertension (11). Anti-ARS antibodies are directed against cytoplasmic enzymes that catalyze the formation of the aminoacyl-tRNA complex from an amino acid and its cognate tRNA (3). Marguerie et al. (12) described a series of $29 \mathrm{PM} / \mathrm{DM}$ patients with anti-ARS antibodies who were more likely to have fevers, dyspnea, mechanic's hands, arthritis and ILD than those without such antibodies. Yoshifuji et al. (10) stratified $74 \mathrm{PM} / \mathrm{DM}$ patients into anti-ARS-positive (including two anti-OJ-positive patients) and -negative groups and found that ILD and mechanic's hand occurred significantly more frequently in the anti-ARS-positive group.

To date, eight antisynthetase antibodies have been identified, and anti-Jo1, anti-PL7 and anti-PL12 antibodies are the strongest markers for ILD; anti-Jo1 antibodies are the most common $(13,14)$. Anti-OJ autoantibodies are a type of antiARS antibodies originally identified after the original sera from "patient OJ" showed significant inhibition of isoleucyltRNA synthetase activity (15). To the best of our knowledge, two reports have been published regarding the clinical significance of anti-OJ autoantibodies in ILD patients with PM/DM $(9,16)$. Additionally, there are seven reports describing the clinical significance of anti-OJ autoantibodies $(9,10,16-20)$. Sato et al. (9) screened 1,135 Japanese patients who had or were suspected of having CTDs and detected seven patients positive for anti-OJ autoantibodies (four with PM, three with IIPs), all of whom had ILD. These results suggested that the presence of anti-OJ autoantibodies is more closely associated with ILD than myositis. Targoff et al. (16) reported the cases of six anti-OJ autoantibody-positive PM/DM patients with ILD; however, he did not describe the details of these cases, including the symptoms observed on first visit and the responsiveness to the therapy. The characteristics of our present two patients are summarized in Table 2, and previous reports and our cases are summarized together in Table 3. Yoshifuji et al. (10) reported that, in anti-ARS autoantibody-positive PM/DM patients, the development of ILD often precedes that of myopathy. Our patients experienced respiratory symptoms that preceded the development of other symp- 
Table 3. Literature Review and Our Cases

\begin{tabular}{|c|c|c|c|c|c|c|}
\hline & Sato et al. ${ }^{9}$ & Targoff et al. ${ }^{16}$ & Friedman et al. $^{20}$ & other reports ${ }^{17-19}$ & our cases & Total \\
\hline No patients & 7 & 9 & 2 & 3 & 2 & 23 \\
\hline Age at onset, mean \pm S.D. years & $53 \pm 18$ & $52 \pm 13$ & 36,50 & $54 \pm 8$ & 67,74 & $53 \pm 11$ \\
\hline No male/no. female & $3 / 4$ & $4 / 5$ & $1 / 1$ & $0 / 3$ & $1 / 1$ & $9 / 14$ \\
\hline \multicolumn{7}{|l|}{ Dignosis } \\
\hline PM/DM with ILD & $3 / 7$ & $6 / 9$ & $0 / 2$ & $0 / 3$ & $2 / 2$ & $11 / 23(47.8 \%)$ \\
\hline PM,RA with ILD & $1 / 7$ & $0 / 9$ & $0 / 2$ & $0 / 3$ & $0 / 2$ & $1 / 23(4.3 \%)$ \\
\hline IIPs & $3 / 7$ & $3 / 9$ & $2 / 2$ & $3 / 3$ & $0 / 2$ & $11 / 23(47.8 \%)$ \\
\hline fever & $3 / 7$ & NA & $0 / 2$ & $0 / 3$ & $1 / 2$ & $4 / 14(2.9 \%)$ \\
\hline ILD & $7 / 7$ & $8 / 9$ & $2 / 2$ & $3 / 3$ & $2 / 2$ & $22 / 23(95.7 \%)$ \\
\hline arthritis & $4 / 7$ & $6 / 7 *$ & $1 / 2$ & $2 / 3$ & $0 / 2$ & $13 / 21(61.9 \%)$ \\
\hline Raynaud's phenomenon & $0 / 7$ & $1 / 7^{*}$ & $0 / 2$ & $1 / 3$ & $0 / 2$ & $2 / 21(9.5 \%)$ \\
\hline myositis & $4 / 7$ & $8 / 9$ & $0 / 2$ & $1 / 3$ & $2 / 2$ & $15 / 23(65.2 \%)$ \\
\hline
\end{tabular}

PM: polymtositis, DM: dermatomyositis, RA: rheumatoid arthritis, IIPs: idiopathic intestitial pneumonias

* a part of data were not available. NA, data not a vailable

The diagnosis of IIPs (idiopathic interstitial pneumonias) was based on consensus classification of IIPs. ${ }^{10}$

toms. On physical examination, neither sclerodactyly, Raynaud's phenomenon nor sicca were found in either patient. Although Raynaud's phenomenon is often associated with the presence of anti-Jo-1 antibodies, it may not occur in patients with anti-OJ antibodies (Table 3).

The response of ILD to glucocorticoid therapy is significantly better in anti-ARS positive PM/DM patients than in anti-ARS negative patients. Three of the four anti-OJ autoantibody-positive ILD patients with PM reported by Sato et al. also responded to glucocorticoid therapy. Both of our patients also showed good clinical responses following treatment with high-dose glucocorticoids without immunosuppressants. There may be a tendency for ILD patients with anti-OJ autoantibodies to show good prognoses and good responses to glucocorticoid monotherapy. Although ILD has a tendency to recur in anti-ARS autoantibodypositive patients (10), in both of the present cases, the ILD remained static since the first treatment.

Performing a histopathological analysis of ILD is often difficult in patients with clinical symptoms due to the risk of deterioration. Although histopathological analyses could not be performed in the present cases, the $\mathrm{CT}$ findings suggested a diagnosis of ILD. To the best of our knowledge, there have been few detailed reports regarding the chest CT findings of ILD in PM/DM patients with anti-OJ autoantibodies. Both of our cases showed similar findings on chest HRCT, including bilateral consolidation and ground glass attenuation with volume loss in the lower lobes and peribronchovascular predominance. Koreeda et al. (17) reported the chest HRCT findings of one anti-OJ autoantibody-positive ILD patient without PM/DM, which included bilateral ground glass opacities, consolidations volume loss in the lung bases and peribronchovascular predominance. These reported findings are similar to the HRCT findings observed in our patients. Such findings may be associated with the presence of anti-OJ autoantibodies.

As described above, there are no reports regarding the detailed radiological and histopathological findings of ILD in
PM/DM patients with anti-OJ autoantibodies; however, some reports have described the details of radiological and histopathological analyses of idiopathic interstitial pneumonia (IIPs) patients with anti-OJ autoantibodies. The results of a previous histopathological analysis of six IIP patients with anti-OJ autoantibodies $(9,16-18)$ revealed an NSIP pattern in two of the six patients, a cryptogenic organizing pneumonia pattern in two patients, an UIP pattern in one patient and no diagnosis in one patient. Unfortunately, in our series, we could not perform video-assisted thoracoscopic surgery (VATS) due to a deterioration of respiratory symptoms. However, the chest HRCT findings were not typical of IPF/ UIP, as in previous reports. The histopathological analysis described above may indicate good responses to steroid therapy in ILD patients with anti-OJ autoantibodies (21).

Our results and those of previous reports suggest that anti-OJ autoantibody-positive PM/DM patients with ILD exhibit distinct features compared to ILD patients with other anti-ARS antibodies. The course of ILD in such patients may show a good response to glucocorticoid therapy and be associated with a good prognosis, as in other anti-ARS antibody-positive patients (10). Lymphocyte-predominant BAL and HRCT findings of bilateral consolidation predominantly distributed along the bronchovascular bundles in both lower lobes and areas with ground glass attenuation and linear opacities may be clinical features of anti-OJ autoantibody-positive PM/DM patients with ILD. In such patients, ILD has a tendency to follow a subacute pattern and not become a chronic disease. Although anti-OJ autoantibody screening tests are not available, except in some research institutes, testing for anti-OJ autoantibodies is very important clinically because it may distinguish subtypes of both PM/DM and anti-ARS syndromes and predict a patient's prognosis. The clinical properties of ILD patients with PM/DM may be classified according to the presence or absence of anti-OJ autoantibodies. Further accumulation and analysis should be conducted to clarify whether anti-ARS autoantibodies truly affect the prognoses of ILD patients 
with PM/DM and distinguish between the differences observed among types of anti-ARS autoantibody-positive ILD.

\section{Author's disclosure of potential Conflicts of Interest (COI).}

Ishida T: Honoraria, Abott, Taisyo-Toyama and Daiichi-Sankyo.

\section{References}

1. Targoff IN. Immune manifestations of inflammatory muscle disease. Rheum Dis Clin North Am 20: 857-880, 1994.

2. Hirakata M, Nagai S. Interstitial lung disease in polymyositis and dermatomyositis. Curr Opin Rheumatol 12: 501-508, 2000.

3. Mathews M, Berstein R. Myositis auto-antibodies inhibits histidyltRNA synthetase: a model for auto-immunity. Nature 83: 304-317, 1983.

4. Hirakata M, Suwa A, Nagai S, et al. Anti-KS: identification of autoantibodies to asparaginyl-transfer RNA synthetase associated with interstitial lung disease. J Immunol 162: 2315-2320, 1999.

5. Betteridge Z, Gunawardena H, North J, Slinn J, McHugh N. Antisynthetase syndrome: a new autoantibody to phenylalanyl transfer RNA synthetase (anti-Zo) associated with polymyositis and interstitial pneumonia. Rheumatology 46: 1005-1008, 2007.

6. Katzap E, Barilla-LaBarca ML, Marder G. Antisynthetase syndrome. Curr Rheumatol Rep 13: 175-181, 2011.

7. Love LA, Leff RL, Fraser DD, et al. A new approach to the classification of idiopathic inflammatory myopathy: myositis-specific autoantibodies define useful homogeneous patient groups. Medicine 70: 360-374, 1991.

8. Suwa A, Hirakata M, Satoh S, Ezaki T, Mimori T, Inada S. A case of polymyositis with anti-OJ (isoleucyl-transfer RNA synthetase) antibodies. Clin Exp Rheumatol 17: 755-756, 1999.

9. Sato S, Kuwana M, Hirakata M. Clinical characteristics of Japanese patients with anti-OJ (anti-isoleucyl-tRNA synthetase) autoantibodies. Rheumatology 46: 842-845, 2007.

10. Yoshifuji H, Fujii T, Kobayashi S, et al. Anti-aminoacyl-tRNA synthetase antibodies in clinical course prediction of interstitial lung disease complicated with idiopathic inflammatory myopa- thies. Autoimmunity 39: 233-241, 2006.

11. Marie I, Hatron PY, Dominique S, et al. Short-term and long-term outcomes of interstitial lung disease in polymyositis and dermatomyositis: a series of 107 patients. Arthritis Rheum 63: 3439-3447, 2011.

12. Marguerie C, Bunn CC, Beynon HL, et al. Polymyositis, pulmonary fibrosis and autoantibodies to aminoacyl-tRNA synthetase enzymes. Q J Med 77: 1019-1038, 1990.

13. Kang EH, Lee EB, Shin KC, et al. Interstitial lung disease in patients with polymyositis, dermatomyositis and amyopathic dermatomyositis. Rheumatology 44: 1282-1286, 2005.

14. Connors GR, Christopher-Stine L, Oddis CV, Danoff SK. Interstitial lung disease associated with the idiopathic inflammatory myopathies: what progress has been made in the past 35 years? Chest 138: 1464-1474, 2010.

15. Targoff IN. Autoantibodies to aminoacyl-tranfer RNA synthetases for isoleucine and glycine. Two additional synthetases are antigenic in myositis. J Immunol 144: 1737-1743, 1990.

16. Targoff IN, Trieu EP, Miller FW. Reaction of anti-OJ autoantibodies with components of the multi-enzyme complex of aminoacyltRNA synthetases in addition to isoleucyl-tRNA synthetase. J Clin Invest 91: 2556-2564, 1993.

17. Koreeda Y, Higashimoto I, Yamamoto M, et al. Clinical and pathological findings of interstitial lung disease patients with antiaminoacyl-tRNA synthetase autoantibodies. Intern Med 49: 361$369,2010$.

18. Shimizu K, Tai H, Kuwano K. A case of cellular NSIP with antiOJ (anti-isoleucyl tRNA synthetase) antibodies. Nihon Kokyuki Gakkai Zasshi 48: 45-48, 2010.

19. Gelpí C, Kanterewicz E, Gratacos J, Targoff IN, RodríguezSánchez JL. Coexistence of two antisynthetases in a patient with the antisynthetase syndrome. Arthritis Rheum 39: 692-697, 1996.

20. Friedman AW, Targoff IN, Arnett FC. Interstitial lung disease with autoantibodies against aminoacyl-tRNA synthetases in the absence of clinically apparent myositis. Semin Arthritis Rheum 26: 459467, 1996.

21. Flaherty KR, Toews GB, Travis WD, et al. Clinical significance of histological classification of idiopathic interstitial pneumonia. Eur Respir J 19: 275-283, 2002.

(C) 2012 The Japanese Society of Internal Medicine http://www.naika.or.jp/imonline/index.html 